\title{
CAN WE PREDICT TREND IN TECHNOLOGY?
}

\author{
Zoran Lovreković \\ Higher Education Technical School of Professional Studies \\ lovrekovic@vtsns.edu.rs
}

Original Scientific Paper

10.5937/jouproman9-32024

\begin{abstract}
This paper shows that the foundations of today 's information technology were laid mainly in the 1960s and 1970s. Even the World Wide Web was founded in the 1990s. Following the philosophy of Moore's Law, and generalizing the findings from the past, it is possible to conclude that, in the same way, the foundations of the technology that will mark the next two decades have already been laid these days. Based on that, predictions that will be technological trends in the next 20 years can be confirmed with great certainty. Further development and ubiquitous application of artificial intelligence, IoT, and 5G networking will certainly fundamentally change our lives.
\end{abstract}

Keywords: Technological predictions, computer networks, Moore's law, artificial intelligence, Augmented reality, spatial web

\section{Introduction}

Do we need psychics to predict future technological trends, or is it possible to predict them using scientific methods? The basis of the idea of how to predict the development of technology in the next 20 years, presented in this paper, is to use looking back, and follow the timeline to the present day, and based on the data thus collected and set in the appropriate context, generate knowledge - what trends in technology development await us in the next 20 years. A similar principle was used by Gordon Moore in defining a rule known today as Moore's Law.

\section{First, let's look back}

On 04.12.2020. died Narinder Sing Kapany. He was an Indo-American physicist, best known for his work on optical fibers. He was considered the "father of optical fibers" and was the creator of the first optical cable. In 1950, at Imperial College, he made a bundle of several thousand optical fibers and demonstrated its success in image transmission. [1]

In 1958, AT\&T introduced the first modem. It worked at $110 \mathrm{bps}$. The main function of this type of modem was to connect SAGE (Semi-Automatic Ground Environment) computers across America (part of the US air defense). With this modem, sending a short text similar to today's email would take about 20 minutes.[2]

The first video game made for a computer is considered to be "SpaceWar". Made by Steve Russell on the MIT PDP1 mainframe computer, in 1962. However, as early as 1952, A.S. Douglas is writing a doctoral dissertation at the University of Cambridge entitled Human-Computer interaction, and is developing a Tic-TacToe computer game [3]

In 1963, Douglas Engelbart constructed the first computer mouse.[4] 


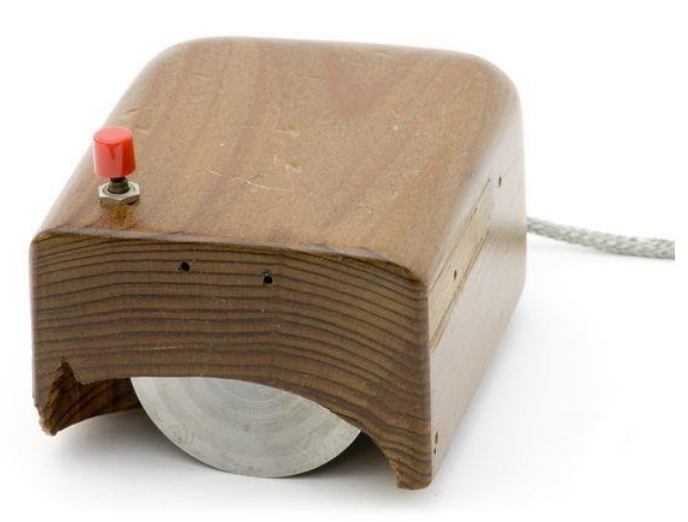

Fig. 1 First Computer Mouse

In 1968, the company INTEL was founded. The founders were Robert Noyce and Gordon Moore. The company name is an abbreviation of "Integrated Electronics". Her first products were single-kilobit DRAM memory chips.[5]

1971 The first computer speech recognition software, IBM's Automatic Call Identification System, appears. [6]

Also in 1971, Ray Tomlinson, who played a key role in the development of
ARPANET (the forerunner of today's Internet), sent the first e-mail [7]

In 1969 ARPANET is formed. The idea for this dates back to 1961, when Leonard Kleinrock wrote a scientific paper entitled "Information Flow in Large Communication Nets."[8] Finally, on October 29, 1969, UCLA's Network Measurement Center, Stanford Research Institute (SRI), University of CaliforniaSanta Barbara, and University of Utah were connected to a computer network. The first message was "LO", which is actually an attempt by student Charles Kline to $\log$ in to the SRI computer from the university. the message is incomplete because the system crashed.

In 1957, the first artificial satellite was launched - Sputnik 1 [9]. The idea of artificial satellites comes from science fiction writer Arthur C. Clarke, who published an article with the idea of artificial satellites in geo-stationary orbit in Wireless World magazine in October 1945.[10] 


\section{EXTRA-TERRESTRIAL RELAYS}

\section{Can Rocket Stations Give World-wide Radio Coverage?} LThough it is possible, by
a suitable choice of fre-
quencies and routes, to provide telephony circuits between any two points or regions of the earth for a large part of the time, long-distance communication is greatly hampered by the peculiarities of the ionosphere, and there are even occasions when it may be impossible. A true broadcast service, giving constant field strength at all times over the whole globe would be invaluable, not to say indispensable, in a world society.

Unsatisfactory though the telephony and telegraph position is, that of television is far worse, since ionospheric transmission cannot be employed at all. The service area of a television station, even on a very good site, is only about a hundred miles across. To cover a small country such as Great Britain would require a network of transmitters, connected by coaxial lines, waveguides or VHF relay links. A recent theoretical study ${ }^{1}$ has shown that such a system would require repeaters at intervals of fifty miles or less. A system of this kind could provide television coverage, at a very considerable cost, over the whole of a small country. It would be out of the question to provide a large continent with such a service, and only the main centres of population could be included in the network.

The problem is equally serious when an attempt is made to link television services in different parts of the globe. A relay chain several thousand miles long would cost millions, and transoceanic services would still be impossible. Similar considerations apply to the provision of wide-band frequency modulation and other services, such as high-speed facsimile which are by their nature restricted to the ultra-high-frequencies.

Many may consider the solution proposed in this discussion too far fetched to be taken very seriously. Such an attitude is unreasonable, as everything envisaged here is a

\section{BY ARTHUR C. CLARKE}

logical extension of developments in the last ten years-in particular the perfection of the long-range rocket of which $\mathrm{V}_{2}$ was the prototype. While this article was being written, it was announced that the Germans were considering a similar project, which they believed possible within fifty to a hundred years.

Before proceeding further, it is necessary to discuss briefly certain fundamental laws of rocket propulsion and "astronautics." A rocket which achieved a sufficiently great speed in flight outside the earh's atmosphere would never return. This "orbital" velocity is $8 \mathrm{~km}$ per sec. ( 5 miles per sec), and a rocket which attained it would become an artificial satellite, circling the world for ever with no expenditure of power-a second moon, in fact. the atmosphere and left to broad cast scientific information back to the earth. A little later, manned rockets will be able to make similar flights with sufficient excess power to break the orbit and return to earth.

There are an infinite number of possible stable orbits, circular and elliptical, in which a rocket would remain if the initial conditions were correct. The velocity of $8 \mathrm{~km} / \mathrm{sec}$, applies only to the closest possible orbit, one just outside the atmosphere, and the period of revolution would be about 90 minutes. As the radius of the orbit increases the velocity decreases, since gravity is diminishing and less centrifugal force is needed to balance it. Fig. I shows this graphically. The moon, of course, is a particular case and would lie on the curves of Fig. I if they were produced. The proif they were produced. The pro-
posed German space-stations

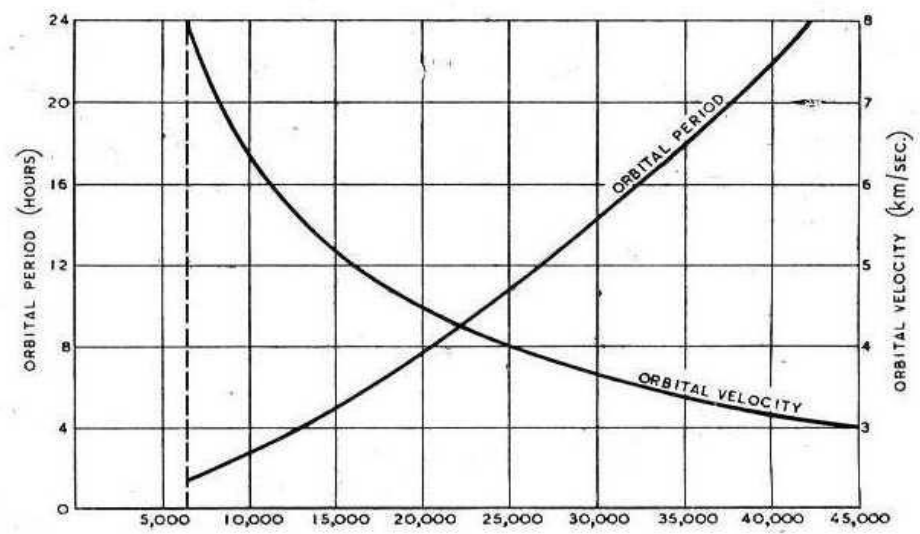

DISTANCE FROM CENTRE OF EARTH. (KILOMETRES)

Fig. 1. Variation of orbital period and velocity with distance from the centre of the earth.

The German transatlantic rocket Aro would have reached more than half this velocity.

It will be possible in a few more years to build radio controlled rockets which can be steered into such orbits beyond the limits of would have a period of about four and a half hours.

It will be observed that one orbit, with a radius of $42,000 \mathrm{~km}$, has a period of exactly 24 hours. A body in such an orbit, if its plane coincided with that of the

Fig.2 Arthur C. Clarke article with idea on artificial satellites in geo-stationary orbit

In 1991 Tim Berners Lee introduces the World-Wide-Web service at CERN [11] 


\section{Technology development curve}

In the early 1970s, in preparation for a conference on the future of memory chips, a Silicon Valley veteran, Dr. Gordon Moore drew on logarithmic paper the capacity of all previous generations of computer memory chips.[12] The chart then continued drawing the development of chips of the same properties (RAM) in the future planned by his company, Intel Corporation. To his surprise, the graph was a straight line and showed that every two years the complexity of memory chips doubles and that this trend will continue. He knew that his company was progressing very fast, but even for him, this kind of chart was surprising. Drawing conclusions based on the described graph, Moore predicted that in just twenty years (until 1991) there would be a jump from the 1Kbit DRAM chip to the 1Mbit Dram chip. In 1971, the idea of having a chip with a capacity of one megabit seemed unrealistic and it seemed impossible that such a chip would be realized. History has shown, however, that Moore was right in his predictions. Moreover, the reality exceeded expectations. According to Denos S. Gazis [13] of the IBM Research Center, IBM is able to quadruple the density of memory chips approximately every three years, with a tendency to speed up this process.

\section{Microprocessor Transistor Counts 1971-2011 \& Moore's Law}

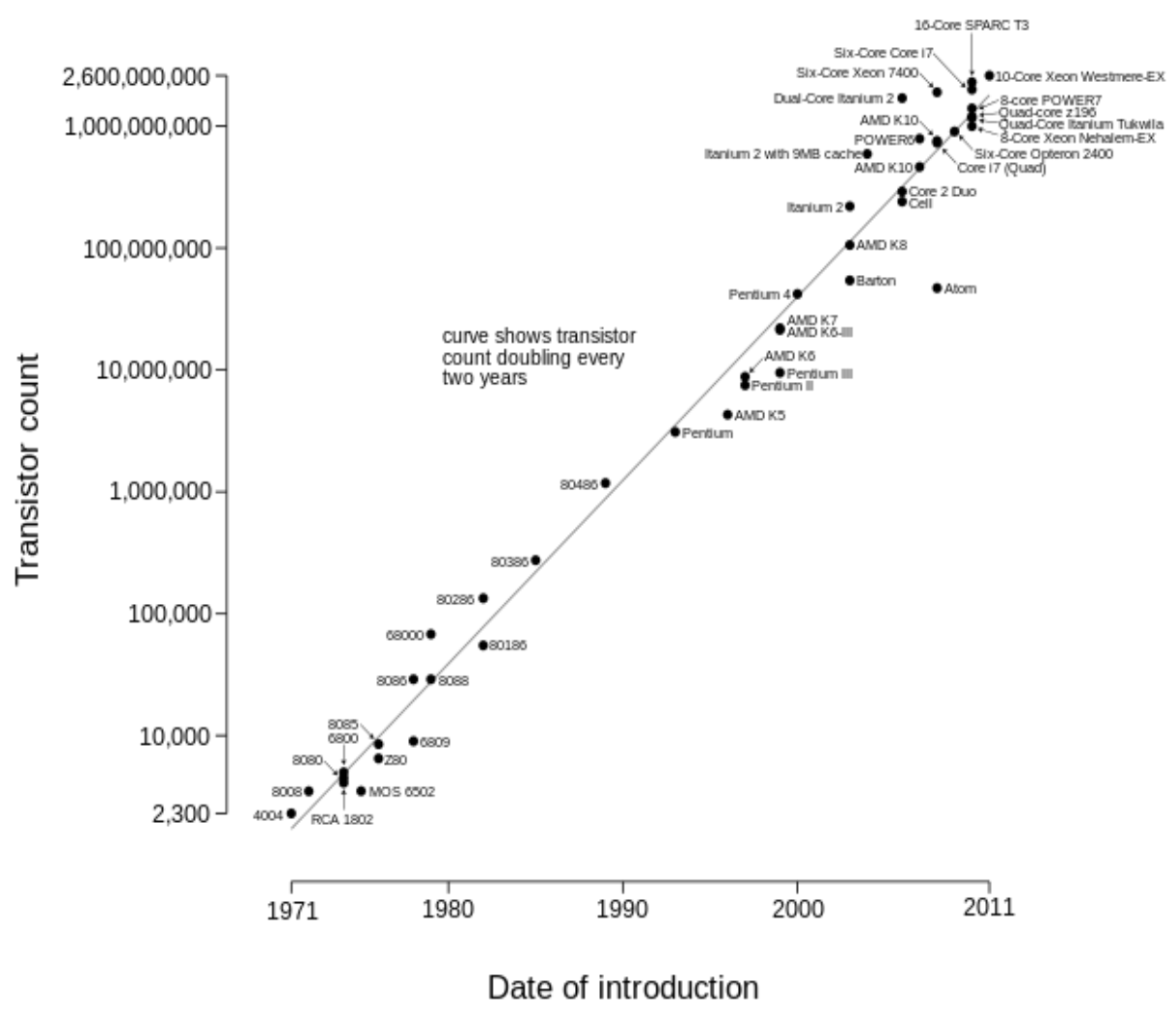

Fig. 2 Moore's Law 
Research has shown that Moore's Law can be applied equally well to other integrated circuits. In fact, it provides a constant vision for the development of the entire electronics industry. From 1947, when Shcokley, Baredeen, and Brattain developed the first transistor, only 45 years passed until an integrated circuit containing more than thirty million transistors and capacitors was produced.

In 1970, when semiconductor memory began to replace massively used memory with magnetic cores, its price was about 5 cents per bit. Twenty years later, the price of this type of memory has dropped to a thousandth of a cent per bit. And every drop in the price of computers and computer equipment becomes a strong impetus for the introduction of new areas and new ways of applying this equipment, as well as the development of more sophisticated, more powerful and simpler software. Kenneth Flamm of the Brookings Institute, following the price and performance of computer equipment between 1957 and 1978, found that the improvement, defined as the price / performance ratio, was at a rate of about $25 \%$ per year, almost linearly, making an improvement of about 1000 times in just two decades. UNIVAC 1, created in 1950, consisted of 5,000 vacuum tubes, and was able to perform 1,000 calculations per second. It was the first commercial computer, made in just six copies, three of which were sold to the U.S. Census Bureau for \$250,000 apiece.[14] In 1991, a computer of the same price, the MIPS6000, the size of a small cabinet, performed 55 million calculations per second. In 2020, PCs perform billions of calculations per second at a cost of the order of $\$ 1,000$. The standard disk capacity is with the same growth trend. In 1961, General Electric drives provided approximately 25 kilobits per square inch, while in 1991 Hitachi launched a disk with a capacity of 151 megabits per square inch. Today's disks have a capacity of several hundred gigabytes per square inch. The power consumption of today's computers is also lower by several orders of magnitude than their ancestors from the fifties and sixties. Today's computers are a million times lighter and also a few orders of magnitude smaller. This enables their installation almost everywhere: - from installations on our tables, to applications in military missiles, or video games and children's toys. The described advances, as well as similar examples of technology development in semiconductor technology, computer hardware, data recording, storage and use, software development, and communications, allow us to guess what the world will look like in twenty or fifty years. The listed technologies are exactly the ones that the US government has declared critically important and which need to be developed with special care in order for the US to maintain its competitiveness at the world level in the next century as well.

\section{Predictions of new trends in technology for the next 20 years:[15], [16], [17]}

- Global gigabit connectivity will be achieved for everyone and everywhere, at an ultra low cost

- The average human life expectancy will increase by 10 years or more

- Augmented reality and spatial web will achieve ubiquitous development: -a combination of augmented reality (web 3.0 and ubiquitous, spatial web) and $5 \mathrm{G}$ networks that will offer connection speeds of $10 \mathrm{~Gb} / \mathrm{s}$, transform our daily lives and make an impact on all industries, sales, advertising, education and entertainment. 
- Everything that is produced will be "smart", with built-in intelligence. The prices of specialized chips with machine learning and microscopic sensors are falling rapidly, and in combination with broadband networks, we are moving towards a decade in which every device will be intelligent. The devices respond to voice commands and anticipate your needs. Intelligent drones watch over and monitor your children. Children's toys remember children's faces and their names.

- Artificial intelligence will reach human intelligence by 2030. Artificial intelligence algorithms and machine learning tools are becoming available, open source, and allow an individual to use such algorithms and devices to improve their cognitive abilities, expand their problem-solving capacities and build new businesses at a price that is a small amount of the current one.

Human-artificial intelligence collaboration is a "rocket flywheel" for all professions. The rise of the artificial intelligence platform as a service (AIaaS) will enable people to have a partner in artificial intelligence in every aspect of their work and life, at every level, in every industry. Support in creative work, generation of new ideas and innovations will become a common area for partnership with artificial intelligence. Somewhere, as in diagnostics in medicine, working without a partnership with artificial intelligence will be unthinkable.

- Autonomous vehicles and flying cars will redefine human travel that will be much faster and cheaper.

- Ability to produce and deliver at the customer's request in an instant period (instant economy of things) with widespread use of robots, drones, 3D printers and artificial intelligence in high individualization and adaptability to each customer in the production and delivery of goods.

- The ability to feel, experience and know everything, in any place, at any time. Over 100 billion sensors (The Internet of Everything) will monitor and sensory (image, video, listening, measuring ...) everywhere, every detail of our environment, all the time. Global satellites, drones, autonomous cars and augmented reality cameras will be part of the global sensor matrix, allowing us to know everything, anywhere and anytime.

- Redundancy of marketing and advertising. As artificial intelligence becomes embedded in everyday life, your personal AI (Artificial Intelligence) assistant will learn and understand what you need, better than yourself. We will trust our AI to make decisions about what to buy better than we would be able to, and our trust in a personal AI shopping assistant will be complete.

- Cellular agriculture is moving from laboratories to the interior of cities, providing high-quality, healthier and cheaper proteins for the population.

- High-bandwidth brain-computer interfaces (BCIs) are entering public use.

- High-resolution virtual reality (VR) transforms retail and real estate.

- The focus on sustainability and environmental protection is increasing

- Gene therapy leads to disease minimization. A wide range of infectious diseases, from AIDS to Ebola, are becoming curable. Gene editing technology is advancing in precision and ease of use, enabling families to be cured of hundreds of inherited genetic diseases. This will be made possible by the convergence of different biotechnologies, genomic sequencing and artificial intelligence. 
- Internet of Things (IOT). IoT are "smart" machines or devices that have a unique identifier and are able to collect and transfer data over the Internet without requiring human-human or humancomputer interaction. They are supported by artificial intelligence and methods for processing huge amounts of data (Big Data). The installation of computer devices in everyday objects increases our standard of living. The field of IoT is comprehensive and wide: Security systems, cars, electronic devices, lights in smart homes and smart commercial environments, public address systems, candy machines, thermostats, etc ...

- Collaborative robots (COBOTS). Humans and robots will work "hand in hand". Today's robots are highly specialized and intelligent enough with high precision to the tasks assigned to them. The future brings better robots, capable of working in pairs with humans.

Intelligent Computer Vision. Big Data and quality optical sensors help machines visually interpret their environment. Face recognition is a great example that computer devices can see. We already use this every day to unlock our mobile phones. Google Lens is also a great example of intelligent machine vision. Visual data can significantly improve future applications.

- Natural language processing will enable machines to understand human language. This will dramatically change the way man interacts with the machine. This technology comes with the development of voice interfaces and chatbots such as Siri, Alexa and Google Assistant. In fact, people are already used to talking to machines.

\section{Conclusion}

Knowledge of software languages and computer hardware components is no longer enough to sustain a country's technological advancement. Artificial intelligence, Fuzzy logic and neural networks will be the basis of every application and every device. The ability to develop the components and applications of artificial intelligence will be a key skill in the near future.

Increasingly faster networks of things, and the mass application of iot and all possible types of sensors, combined with artificial intelligence, will completely redefine our way of doing business, entertainment and life in general.

Robots will no longer be machines for us, but co-workers and colleagues.

A robot that dreams, that has emotions? Science Fiction? Remember Arthur Clark and satellites in geostationary orbit.

\section{References:}

1. A New Method of transporting Optical Images without Aberrations. A. C. S. VAN HEEL. Nature volume 173, page39, 1954

2. https://historycomputer.com/ModernComputer/Basis/modem.htm 1

3. https://www.thoughtco.com/history-ofcomputer-and-). video-games-4066246

4. https://www.computerhistory.org/revolution/inputoutput/14/350

5. https://www.britannica.com/topic/Intel

6. https://www.condecosoftware.com/blog/ahistory-of-voice-recognition-technology/ 
7.

https://www.guinnessworldrecords.com/news/60at 60/2015/8/1971 -first-ever-email-392973

8. https://www.livescience.com/20727-internethistory.html

9. https://www.svetnauke.org/21701-sputnik-1pocetak-kosmicke-ere

10.

http://lakdiva.org/clarke/1945ww/1945ww_305.jpg

11. https://www.livescience.com/20727-internethistory.html

12. (Davidow W. H., Uttal, B., "Total Customer Service", Harper \&Row, New York, 1989., p.p.7678)
13. Gazis, D., C., "Brief Time, Long March" u "Technology 2001", MIT Press, Boston, 1991, page 44.

14. Creating the Computer: Government, Industry, and High Technology. By Kenneth Flamm. Washington, DC: Brookings Institution, 1988. Pp. xi, 282

15.https://singularityhub.com/2020/01/10/20-techmetatrends-to-look-out-for-in-the-2020s/

16. https://www.datacenters.com/news/top-10technology-trends-for-2020-and-the-comingdecade

17. https://duexpress.in/twenty-technology-trendsthat-will-define-the-next-decade/ 